\title{
TECNOLOGIA EDUCATIVA PARA O AUTOCUIDADO DE PACIENTES SUBMETIDOS AO DRENO TORÁCICO
}

\author{
EDUCATIONAL TECHNOLOGY FOR THE SELF-CARE OF PATIENTS SUBMITTED TO THO- \\ RACIC DRAINAGE
}

\author{
Iana Bruna Parente Cardoso ${ }^{a^{*}}$, Daliane Ferreira Marinho ${ }^{b^{*}}$ \\ ianabruna95@gmail.com ${ }^{\mathrm{a}}$, dalianemarinho@yahoo.com.br ${ }^{\mathrm{b}}$ \\ Universidade do Estado do Pará
}

\section{RESUMO}

Data de Submissão: 14/03/2019

Data de Aceite: 21/10/2019

Introdução: A utilização de instrumentos educativos na área da saúde é prática comum no Sistema Único de Saúde, levando a alterações positivas no quadro do paciente. Objetivos: O objetivo da pesquisa foi elaborar e validar uma cartilha para o autocuidado de pacientes submetidos ao dreno torácico. Materiais e métodos: $\mathrm{O}$ estudo foi desenvolvido em duas fases por meio de abordagem quantitativa. Na primeira ocorreu a confecção da cartilha após uma revisão de literatura. Já na segunda ocorreu a validação por meio da avaliação de 13 juízesespecialistas e de 13 representantes do público-alvo. Esta avaliação se deu através de questionários com questões que precisavam alcançar valor de concordância de $80 \%$. A pesquisa foi realizada após aprovação do Comitê de Ética em Pesquisa. Resultados: Após a revisão de literatura a cartilha intitulada "Dreno de tórax: O que fazer?" foi criada contendo tópicos importantes sobre o assunto, passando pela análise dos juízes-especialistas obtendo concordância de $99,18 \%$, sendo o primeiro bloco de perguntas com 100\%, o segundo $100 \%$ e o terceiro $96,15 \%$. Apesar de todos receberem valor maior que $80 \%$, houve uma sugestão de modificação que foi acatada passando, então, para a avaliação do público-alvo. Pela qual se constatou concordância de $100 \%$, com todos os blocos recebendo $100 \%$, sem sugestões. Conclusão: A cartilha foi considerada válida pelos 26 juízes que a avaliaram. Espera-se que a mesma seja utilizada na prática e possa promover o conhecimento sobre os cuidados necessários para melhorar a qualidade de vida dos pacientes.

Palavras-chave: Estudos de validação; derrame pleural; autocuidado.

\begin{abstract}
Introduction: The use of educational printed instruments in the health area is a common practice in the Unified Health System, leading to positive changes in the patient's condition. Objectives: The objective of the research was to develop and validate an booklet for the self-care of patients submitted to thoracic drainage. Materials and methods: The study was developed in two phases through a quantitative approach. In the first the preparation of the booklet occurred after a literature review. Already in the second phase, validation took place through the evaluation of 13 specialist judges and 13 representatives of the target audience. This evaluation was done through questionnaires with questions that had to reach an agreement value of $80 \%$. The research was carried out after approval of the Research Ethics Committee. Results: Following the literature review, the booklet entitled "Chest drain: What to do?" Was created containing important topics on the subject, passing the analysis of the expert judges obtaining general agreement of $99.18 \%$, being the first block of questions with $100 \%$, the second $100 \%$ and the third $96,15 \%$. Although all received a value greater than $80 \%$, there was a suggestion of modification that was accepted so that the instrument passed to the evaluation of the target audience. By which a general agreement of $100 \%$ was verified, with all the blocks receiving $100 \%$, without suggestions. Conclusion: The booklet was considered valid by the 26 judges who evaluated it. It is expected that it will be used in practice and can promote the knowledge about care needed to improve the quality of life of patients.
\end{abstract}

Keywords: Validation studies; pleural effusion; self-care. 


\section{Introdução}

A utilização de instrumentos educativos impressos na área da saúde, como cartilhas, folhetos e manuais, é prática comum no Sistema Único de Saúde (SUS) e são imprescindíveis, pois possuem capacidade de gerar resultados expressivos para os sujeitos das atividades educativas, levando a alterações positivas no quadro do paciente ${ }^{1},{ }^{2}$.

Este estudo trata, então, da construção e validação de uma cartilha de orientação destinada aos pacientes submetidos ao dreno torácico visto que são muitas as doenças que podem atingir o sistema respiratório podendo gerar prejuízos na mecânica respiratória ${ }^{2,3}$.

O derrame pleural é uma dessas doenças, trata-se se do acúmulo de líquido no espaço pleural, sendo uma patologia comum na prática clínica e responsável por um número elevado de internações. Uma das suas formas de tratamento é por meio da drenagem torácica, em que um dreno é introduzido por meio da parede do tórax na cavidade pleural com o intuito de permitir a saída dos líquidos ou gases presentes nesse espaço, mantendo ou restaurando a pressão negativa no local e auxiliando na reexpansão do pulmão colapsado, após cirurgia, traumas ou afecções clínica $^{3,4}$.

Embora esse procedimento seja frequente em hospitais, o que tem se observado na prática clínica é que os cuidados com os drenos torácicos costumam variar, não só de um local para outro, como também entre os componentes da equipe multiprofissional que atendem ao paciente com dreno torácico, o que pode modificar o resultado com a conduta, pois a manipulação inadequada desse dreno pode gerar inúmeras complicações, como infecções e traumas em órgãos próximos, levando a um aumento do tempo de hospitalização e podendo ocasionar até mesmo a morte ${ }^{4}$.

Em hospitais da rede pública do município de Santarém é possível observar o elevado índice de pacientes que são submetidos a este tipo de dreno, principalmente devido aos altos números de lesões traumáticas de tórax, ocasionados em sua maioria por acidentes com armas brancas e de fogo. Com base nisso, esse estudo teve como objetivo elaborar e validar uma cartilha para o autocuidado de pacientes submetidos ao dreno torácico.

\section{Metodologia}

O estudo utilizou abordagem quantitativa, sendo dividido em duas fases: Produção e Validação da cartilha. Obedeceu a Resolução ${ }^{\circ}$ 466/12 do Conselho Nacional de Saúde, sendo desenvolvida no ano de 2017, após recebimento do parecer de No 1.914.669 do Comitê de Ética em Pesquisa (CEP) da Universidade do Estado do Pará (UEPA). Além disso, recebeu a autorização do Hospital Municipal de Santarém (HMS), onde a pesquisa foi realizada, e dos voluntários após a assinatura do Termo de Consentimento Livre e Esclarecido (TCLE).

$\mathrm{Na}$ fase de produção ocorreu a confecção da cartilha após uma revisão da literatura, utilizando os artigos dos últimos 5 anos relacionados com orientações e exercícios fisioterapêuticos para pacientes submetidos ao dreno torácico. A partir disso foi realizada a avaliação da mesma por meio da validação do conteúdo, realizada pelos juízesespecialistas e a validação aparente, realizada pelo público-alvo ${ }^{5}$. Utilizamos o valor de concordância de $80 \%$, valor comum em outras pesquisas ${ }^{6,7}$.

Fizeram parte da validação 13 juízesespecialistas e 13 representantes do público-alvo. Entre estes juízes-especialistas estavam 9 juízes da área da saúde (3 enfermeiros, 3 médicos e 3 fisioterapeutas que trabalhavam no HMS) e 4 juízes de outras áreas (2 designer gráfico e 2 pedagogos).

Os juízes da área da saúde deveriam ser especialistas ou estar se especializando na área respiratória, cirúrgica, intensivista ou traumatoortopédica elou ter pelo menos um ano de experiência na área hospitalar. Já os juízes de outras áreas deveriam ter no mínimo um ano de experiência na área de atuação.

Já em relação à avaliação aparente, os 13 indivíduos deveriam estar internados no setor de clínica cirúrgica do HMS e submetidos ao dreno torácico por no mínimo 48 horas, ser maior de 18 anos, estar consciente e orientado no tempo e espaço, ser alfabetizado e aceitar participar da pesquisa.

Foram excluídos os que não se encaixaram nos critérios acima, os pacientes com alta hospitalar, os que apresentem piora do quadro clínico, os que tenham condições clínicas graves e que não 
estejam possibilitados de participar da pesquisa ou ainda aqueles que sejam transferidos.

Os avaliadores responderam um questionário através da utilização da escala de Likert, constituído por questões com as seguintes pontuações: 1 igual a totalmente adequado (TA), 2 igual a adequado (A), 3 igual a parcialmente adequado (PA) e 4 igual a inadequado $(\mathrm{I})^{8}$.

Para a análise quantitativa da cartilha foi utilizada uma representação das repostas da seguinte forma: respostas 1 e 2, significaram pontuação +1 ; respostas 3 , representavam pontuação 0 e respostas 4, estavam relacionadas com pontuação -1. Dessa forma, as respostas poderiam variar de $-1 \mathrm{a}+1$ e quanto mais alto, maior seria o índice de concordância entre eles de que o tópico era relevante.

As respostas foram pontuadas e somadas, gerando uma pontuação para identificar o grau de concordância entre estas, se a concordância alcançasse valor superior a $80 \%$ a cartilha não seria modificada. No entanto, se o valor fosse inferior a mesma passaria por alterações.

Os dados numéricos foram armazenados no programa Microsoft Excel Office 2010, depois analisados no programa Biostat versão 5.0, através de estatística descritiva e os resultados foram organizados em tabelas.

\section{Resultados}

A partir da revisão de literatura, foi criada uma cartilha denominada de "Dreno de tórax: $\mathrm{O}$ que fazer?", contendo as principais orientações e exercícios respiratórios que beneficiam pacientes submetidos ao dreno torácico, surgindo tópicos desde conceitos básicos até importância da reabilitação pulmonar com fisioterapeuta após a alta hospitalar.

Após isso, foi realizada a elaboração textual, utilizando um modelo de elaboração de materiais impressos em saúde, com recomendações quanto a linguagem, layout e ilustração ${ }^{9}$, empregando linguagem acessível a todos os níveis de instrução. Além disso, foram confeccionadas imagens para auxiliar no entendimento das ideias principais e diagramação com a ajuda de um design gráfico.
A cartilha contem 21 páginas mais a capa e o verso e 20 ilustrações, sendo impressa em preto e branco no papel A4 em modo paisagem, sendo cada folha dividida ao meio e acoplando duas páginas. Excetuando-se a capa e o verso que foram impressos coloridos e em papel fotográfico.

Após a elaboração do material, o mesmo passou pela validação dos juízes-especialistas, com o intuito de adequar o conteúdo ao contexto de trabalho dos mesmos ${ }^{10,11}$. As respostas obtidas dos questionários dos juízes-especialistas foram analisadas de forma quantitativa e expostas na Tabela 1, de acordo com a ordem do questionário e a sequência dos três blocos (1. Objetivos; 2. Estrutura e apresentação; e 3. Relevância) dos itens avaliados. 
Tabela 1 - Ordenamento das respostas dos juízes especialistas segundo os blocos e índice percentuais de concordância de cada item.

\begin{tabular}{llllll}
\hline ITENS & \multicolumn{7}{l}{ SCORES (13) } & & \\
\hline BLOCO 1. OBJETIVOS DA CARTILHA & TA & A & PA & I & $\begin{array}{l}\text { Índice de concordância } \\
\text { por bloco }\end{array}$ \\
$\begin{array}{l}\text { 1.1 As informações são importantes para o autocuidado } \\
\text { de pacientes submetidos ao dreno torácico? }\end{array}$ & 12 & 1 & 0 & 0 & 0,100 \\
$\begin{array}{l}1.2 \text { Convida a mudanças de atitudes nestes pacientes? } \\
\text { 1.3 Pode circular no meio científico? }\end{array}$ & 12 & 1 & 0 & 0 & 0,100 \\
$\begin{array}{l}1.4 \text { Atende aos objetivos da atenção à saúde no } \\
\text { autocuidado? }\end{array}$ & 10 & 3 & 0 & 0 & 0,100 \\
\hline Subtotal & 12 & 1 & 0 & 0 & 0,100 \\
\hline Percentual por bloco & 46 & 6 & 0 & 0 & \\
\hline
\end{tabular}

\section{BLOCO 2. ESTRUTURA E APRESENTAÇÃO}

2.1 A cartilha é apropriada para os pacientes?

2.2 As mensagens estão objetivas?

2.3 As informações estão cientificamente corretas?

$2.4 \mathrm{O}$ material está apropriado a pacientes de qualquer nível sociocultural?

2.5 Há uma sequência lógica de conteúdo?

2.6 As informações estão bem estruturadas em concordância e ortografia, numa linguagem acessível aos pacientes?

2.7 A escrita está de fácil compreensão?

2.8 As informações da capa e sumário são coerentes?

2.9 O tamanho do título e dos tópicos estão adequados?

2.10 As ilustrações estão expressivas, servindo para complementar os textos?

2.11 O número de páginas está adequado?

Subtotal

Percentual por bloco

BLOCO 3. RELEVÂNCIA

3.1 Os temas retratam aspectos-chave?

$3.2 \mathrm{O}$ material permite a transferência do aprendizado a diferentes contextos?

3.3 A cartilha propõe a construção de conhecimento?

3.4 O material aborda os assuntos necessários para o saber destes pacientes?

\begin{tabular}{lllll}
\hline Subtotal & 47 & 3 & 2 & 0 \\
\hline Percentual por bloco & & & & $\mathbf{9 6 \%}$ \\
\hline Total & 221 & 24 & 2 & 0 \\
PERCENTUAL TOTAL & & & & \\
\hline
\end{tabular}

TA: Totalmente Adequado. A: Adequado. PA: Parcialmente Adequado. I: Inadequado.

Fonte: Dados da pesquisa (2017). 
Logo em seguida foi realizada a avaliação do público-alvo a fim de verificar a clareza, compreensão e relevância da cartilha. As respostas obtidas dos questionários dos cuidadores foram analisadas de forma quantitativa, expostas na
Tabela 2, de acordo com a ordem do questionário e a sequência dos cinco blocos (1. Objetivos; 2 . Organização; 3. Estilo da escrita; 4. Aparência; e 5. Motivação) dos itens avaliados.

Tabela 2 - Ordenamento das respostas dos pacientes segundo os blocos do questionário e índice percentuais de concordância de cada item.

\begin{tabular}{|c|c|c|c|c|c|}
\hline ITENS & & $\overline{\mathrm{SCO}}$ & $\overline{S(13}$ & & \\
\hline BLOCO 1. OBJETIVOS & TA & A & PA & I & $\begin{array}{l}\text { Índice de concordância } \\
\text { por bloco }\end{array}$ \\
\hline 1.1 A cartilha atendeu aos seus objetivos de orientação? & 9 & 4 & 0 & 0 & 0,100 \\
\hline $\begin{array}{l}\text { 1.2 Ajuda a você ter uma melhor qualidade de vida, } \\
\text { nesse momento? }\end{array}$ & 10 & 3 & 0 & 0 & 0,100 \\
\hline $\begin{array}{l}1.3 \text { A cartilha está adequada para ser usado por você } \\
\text { nesse momento? }\end{array}$ & 9 & 4 & 0 & 0 & 0,100 \\
\hline Subtotal & 28 & 11 & 0 & 0 & \\
\hline Percentual por bloco & & & & & $100 \%$ \\
\hline \multicolumn{6}{|l|}{ BLOCO 2. ORGANIZAÇÃO } \\
\hline 2.1 A capa é atraente e indica o conteúdo da cartilha? & 10 & 3 & 0 & 0 & 0,100 \\
\hline $\begin{array}{l}2.2 \text { O tamanho do título e do conteúdo nos tópicos está } \\
\text { adequado? }\end{array}$ & 11 & 2 & 0 & 0 & 0,100 \\
\hline $\begin{array}{l}2.3 \text { Os tópicos estão em sequência que facilite o } \\
\text { entendimento? }\end{array}$ & 11 & 2 & 0 & 0 & 0,100 \\
\hline $\begin{array}{l}\text { 2.4 Há coerência entre as informações da capa, sumário } \\
\text { e/ou apresentação? }\end{array}$ & 10 & 3 & 0 & 0 & 0,100 \\
\hline 2.5 O material (papel) está apropriado? & 12 & 1 & 0 & 0 & 0,100 \\
\hline 2.6 O número de páginas está adequado? & 12 & 1 & 0 & 0 & 0,100 \\
\hline 2.7 Os temas retratam aspectos importantes? & 12 & 1 & 0 & 0 & 0,100 \\
\hline Subtotal & 78 & 13 & 0 & 0 & \\
\hline Percentual por bloco & & & & & $100 \%$ \\
\hline \multicolumn{6}{|l|}{ BLOCO 3. ESTILO DA ESCRITA } \\
\hline 3.1 A escrita está facilitando o entendimento? & 12 & 1 & 0 & 0 & 0,100 \\
\hline $3.2 \mathrm{O}$ texto é interessante? & 11 & 2 & 0 & 0 & 0,100 \\
\hline $3.3 \mathrm{O}$ vocabulário é de fácil entendimento? & 11 & 2 & 0 & 0 & 0,100 \\
\hline $\begin{array}{l}\text { 3.4 Há associação do tema de cada sessão ao texto } \\
\text { presente? }\end{array}$ & 11 & 2 & 0 & 0 & 0,100 \\
\hline $3.5 \mathrm{O}$ texto está claro? & 11 & 2 & 0 & 0 & 0,100 \\
\hline $\begin{array}{l}3.6 \text { O estilo da redação corresponde ao nível do seu } \\
\text { conhecimento? }\end{array}$ & 11 & 2 & 0 & 0 & 0,100 \\
\hline Subtotal & 67 & 11 & 0 & 0 & \\
\hline Percentual por bloco & & & & & $100 \%$ \\
\hline \multicolumn{6}{|l|}{ BLOCO 4. APARÊNCIA } \\
\hline 4.1 As páginas parecem organizadas? & 13 & 0 & 0 & 0 & 0,100 \\
\hline 4.2 As ilustrações são de fácil entendimento? & 13 & 0 & 0 & 0 & 0,100 \\
\hline
\end{tabular}




\begin{tabular}{|c|c|c|c|c|c|}
\hline \multirow{2}{*}{$\begin{array}{c}\text { ITENS } \\
4.3 \text { As ilustrações servem para complementar os textos? }\end{array}$} & \multicolumn{4}{|c|}{ SCORES (13) } & \multirow[b]{2}{*}{0,100} \\
\hline & 13 & 0 & 0 & 0 & \\
\hline 4.4 As ilustrações estão expressivas e suficientes? & 13 & 0 & 0 & 0 & 0,100 \\
\hline Subtotal & 52 & 0 & 0 & 0 & \\
\hline Percentual por bloco & & & & & $100 \%$ \\
\hline \multicolumn{6}{|l|}{ BLOCO 5. MOTIVAÇÃO } \\
\hline 5.1 A cartilha é adequada para você? & 10 & 3 & 0 & 0 & 0,100 \\
\hline 5.2 Os conteúdos se apresentam de forma lógica? & 11 & 2 & 0 & 0 & 0,100 \\
\hline $\begin{array}{l}\text { 5.3 A cartilha aborda os assuntos necessários para o seu } \\
\text { dia-a-dia? }\end{array}$ & 12 & 1 & 0 & 0 & 0,100 \\
\hline $\begin{array}{l}5.4 \text { Instiga a mudanças de atitudes durante a } \\
\text { internação? }\end{array}$ & 11 & 2 & 0 & 0 & 0,100 \\
\hline 5.5 A cartilha propõe conhecimentos para você? & 10 & 3 & 0 & 0 & 0,100 \\
\hline Subtotal & 54 & 11 & 0 & 0 & \\
\hline Percentual por bloco & & & & & $100 \%$ \\
\hline TOTAL & 279 & 46 & 0 & 0 & \\
\hline PERCENTUAL TOTAL & & & & & $100 \%$ \\
\hline
\end{tabular}

TA: Totalmente Adequado. A: Adequado. PA: Parcialmente Adequado. I: Inadequado. Fonte: Dados da pesquisa (2017).

\section{Discussão}

Validação de conteúdo é a análise das informações contidas em um instrumento, com o intuito de averiguar se os pontos propostos formam uma amostra importante da temática que se pretende medir. Esses instrumentos passarão por uma apreciação detalhada feita por especialistas no assunto, que poderão realizar correções ${ }^{12}$.

Esta validação foi realizada pelos juízesespecialistas, sendo exposto neste artigo na tabela 1. Nessa etapa as respostas foram consideradas válidas, pois obtiveram concordância maior do que $80 \%$. Para calcular o percentual por coluna de escore (TA, A, PA, I) multiplica-se o subtotal de um escore por 100 e divide-se pela soma dos mesmos.

Conforme a Tabela 1, houve propensão a respostas concordantes entre os juízes para valoração TA: $221(89,47 \%)$ e A: $24(9,71 \%)$, sendo que as duas foram somadas pois segundo a escala de Likert, respostas TA e A devem receber valor +1 , significando concordância, ou seja, a cartilha recebeu uma aprovação de 99,18\%. Então, pode-se deduzir que não houve elucidação de discordância, pois dos 19 itens apenas 2 obtiveram alguma resposta PA e nenhuma I.

$\mathrm{Na}$ literatura não há nenhuma pesquisa relacionada à confecção e validação de cartilhas para pacientes acometidos com patologias respiratórias, porém valores semelhantes ao encontrado na presente pesquisa foram encontrados no estudo de Nascimento ${ }^{8}$. Neste estudo a primeira versão do instrumento foi avaliada por 15 juízes-especialistas, havendo uma propensão a respostas para valoração TA $192(59,25 \%)$ e A 95 (29,32\%), atingindo uma concordância de $99,98 \%$.

BLOCO 1: Objetivo- tópico referente ao propósito que se deseja alcançar com a cartilha, pois só se pode dizer que um instrumento é confiável quando suas medidas refletem as medidas reais do atributo investigado ${ }^{10,12}$. Ao analisar a valoração atribuída pelos juízes para este bloco, verificou-se que: $46(88,46 \%)$ para TA, $6(11,54 \%)$ para $\mathrm{A}, 0$ (0\%) para PA e $0(0 \%)$ para I. De acordo com as opções de respostas, das 52 (100\%), todas foram para TA e A, conferindo a aceitação dos itens.

Corroborando com este achado, a pesquisa de Galdino ${ }^{13}$ que validou uma cartilha de autocuidado para pés de pessoas com diabetes, foi verificado 
que nenhum item foi julgado inadequado quanto a este bloco do questionário, o que conferiu uma concordância de $97 \%$ quanto aos objetivos.

Apesar disso, dois dos juízes sugeriram que a cartilha não explicasse somente sobre o acúmulo de líquido no espaço pleural, mas também sobre o acúmulo de outras substâncias, como pus (empiema), ar (pneumotórax) e sangue (hemotórax). Sendo, então ampliado o conceito com o objetivo de englobar mais pacientes.

Esta alteração foi em concordância com a pesquisa de Lima et $\mathrm{al}^{14}$, que criou e validou uma cartilha para prevenção da transmissão vertical do vírus da imunodeficiência humana, e que também adaptou o instrumento de acordo com às sugestões dos juízes, afirmando que este processo é essencial para tornar o material mais completo possível.

BLOCO 2: Estrutura e Apresentação- trata-se de aspectos como organização geral, formatação, coerência e ilustrações. Esta análise tem como objetivo estabelecer a adequação e conformidade dos atributos em questão ${ }^{15}$. Neste aspecto, as respostas dos juízes foram: $128(89,51 \%)$ para TA, $15(10,49 \%)$ para A, $0(0 \%)$ para PA e $0(0 \%)$ para I. Das 143 (100\%) respostas obtidas, todas foram para TA e A, indicando que a cartilha está válida quanto este bloco.

Diferente destes resultados, a pesquisa de Nascimento $^{8}$ demonstra que este bloco de estrutura e apresentação recebeu o menor valor de concordância, sendo de $82,75 \%$. O autor afirma que este processo foi a parte mais complexa devido o seu pouco conhecimento sobre aspectos como: diagramação, linguagem, seleção das fotos e dos títulos.

De acordo com Moreira, Nóbrega e Silva ${ }^{9}$, todo material impresso deve considerar a linguagem, o layout e as ilustrações, pontos que foram levados em consideração neste bloco do questionário. Quanto a linguagem, na presente pesquisa optouse por utilizar palavras simples, evitando termos científicos. Similar a pesquisa de Nascimento ${ }^{8}$, que utilizou dos mesmos artifícios e afirmou que a linguagem das cartilhas deve ser cuidadosa para que todos possam compreender as informações.

Já em relação ás ilustrações e ao layout, a pesquisa optou por selecionar ambos de maneira que tornasse a cartilha mais atrativa facilitando a interpretação do conteúdo. Indo em concordância com outras pesquisas que afirmam que tanto as ilustrações quanto o layout são essenciais para a compreensão do texto ${ }^{8,9}$.

BLOCO 3: Relevância- indica o grau de importância da cartilha. Segundo Oliveira ${ }^{7}$ materiais educativos no contexto da saúde contribuem na prevenção de complicações e no desenvolvimento de habilidades nos pacientes. Neste bloco, as respostas foram: 47 (90,38\%) para TA, $3(5,77 \%)$ para A, $2(3,85 \%)$ para PA e 0 (0\%) para I. Portanto, das 52 (100\%) opções de respostas, $50(96,15 \%)$ foram TA e A, confirmando a validação quanto à relevância.

Resultado semelhante ao encontrado por Oliveira $^{7}$ que se trata da criação e validação de uma cartilha para mulheres mastectomizadas, em que neste bloco das 70 opções de respostas, 64 $(91,42 \%)$ foram para TA e A.

Notamos, então, que a cartilha criada foi considerada como relevante com possibilidade de otimizar o atendimento dos pacientes submetidos ao dreno torácico. Diante disso, concluímos a avaliação realizada pelos juízes-especialistas, passando para avaliação do público-alvo.

Esta segunda fase se trata da validação aparente e tem como objetivo identificar como o público a qual a cartilha é destinada compreende os itens ${ }^{15}$. Os dados obtidos foram expostos na tabela 2, havendo propensão a respostas concordantes para valoração TA: 279 (85,85\%) e A: 46 (14,15\%), sem respostas PA (0\%) e nem I (0\%). Ou seja, a cartilha recebeu aprovação de $100 \%$ por parte destes avaliadores nos cinco blocos analisados, alcançando o grau de significância de validação.

Dado similar à pesquisa de Nascimento ${ }^{8}$, em que após a análise do público-alvo, todos os itens receberam valoração TA (380) e A (123), uma minoria de respostas para PA (16) e nenhuma resposta para I (0), tendo os percentuais de concordância entre os blocos variando entre $95,55 \%$ a $100 \%$, sendo considerada válida.

Além disso, nesta validação aparente os avaliadores não realizaram sugestões, isto pode ter ocorrido devido a baixa escolaridade por parte deste público somado ao baixo nível de conhecimento sobre dreno torácico. Indo em concordância, uma pesquisa de validação realizada por Gentil ${ }^{15}$ detectou que durante 
validação aparente apenas um paciente questionou itens da cartilha sendo este o com maior grau de escolaridade entre os pacientes (pós-graduação).

Diante disso, podemos concluir que o conhecimento é um fator que interfere na saúde pois o mesmo permite que os sujeitos se tornem mais capacitados para assumir o controle da sua saúde, trazendo mudanças no comportamento de risco. Sendo a cartilha um excelente modo de promover hábitos de vida saudáveis. Nesse contexto, surge a cartilha "Dreno de tórax: O que fazer?" validada tanto pelo conteúdo quanto pela aparência, tratando-se de uma pesquisa inédita direcionada para este tipo de público.

\section{Conclusão}

Ao final desta pesquisa, concluímos que o objetivo geral proposto foi alcançado, visto que uma cartilha, para o autocuidado de pacientes submetidos ao dreno torácico, foi elaborada e validada, cujo título é "Dreno de tórax: O que fazer?". Para cria-la foi realizada uma revisão de literatura buscando os cuidados e exercícios respiratórios mais apropriados para este tipo de paciente.

A participação dos juízes-especialistas na validação possibilitou o aprimoramento da tecnologia, alcançando um grau de concordância de $99,18 \%$. Já a participação do público-alvo possibilitou verificar que a mesma está adequada, sendo considerado relevante com um grau de concordância de $100 \%$.

Portanto, espera-se que a cartilha "Dreno de tórax: O que fazer?" possa ser utilizada na prática e promover o conhecimento sobre os autocuidados necessários para melhorar a qualidade de vida dos pacientes, além de servir como embasamento científico para outras pesquisas na mesma área de conhecimento.

\section{Referências}

1. Rebert LM, Hoga LAK, Gomes ALZ. O processo de construção de material educativo para a promoção da saúde da gestante. Rev. Latino-Am. Enfermagem 2012; 20(1).

2. Liebano RE, Hassen MAS, Racy HHMJ, Côrrea, JB. Principais manobras cinesioterapêuticas manuais utilizadas na fisioterapia respiratória: descrição das técnicas. Rev. Ciênc. Méd., Campinas 2009;18(1), 35-45.

3. Pryor Z,Webber A. Fisioterapia para problemas respiratórios e cardíacos. Ed. 4ª, Rio de Janeiro: Guanabara Koogan, 2008.

4. Lima JDR. Rotina de cuidados com dreno de tórax. Grupo hospitalar Conceição, 2013.

5. Clark-carter D. Investigación Cuantitativa en Psicología: Del diseño experimental al reporte de investigación. México: Oxford University Press, 2002.

6. Fernandes MVL, Lacerda RA, Hallage NM. Indicadores de avaliação de práticas de controle e prevenção de infecção do trato urinário associado a cateter: construção e validação. Acta Paul Enferm 2006; 19(2): 174-89.

7. Oliveira MS. Autocuidado da mulher na reabilitação da mastectomia: estudo de validação de aparência e conteúdo de uma tecnologia educativa. [Dissertação Mestrado]. Universidade Federal do Ceará, Fortaleza; 2006.

8. Nascimento MHM. Tecnologia para mediar o cuidareducando no acolhimento de "familiares cangurus" em unidade neonatal: Estudo de Validação. [Dissertação Mestrado]. Universidade do Estado do Pará, Belém; 2012.

9. Moreira MF, Nobrega MML, Silva MIT. Comunicação escrita: Contribuição para a elaboração de material educativo em saúde. Revista Brasileira Enfermagem 2003; 56(2): 184-8

10. Echer IC. Elaboração de manuais de orientação para o cuidado em saúde. Rev. Latino-Am. Enferm. 2005; 13(5) 754-757.

11. Pereira CR. Construção e validação de uma cartilha de orientação sobre o tratamento quimioterápico. [Dissertação Mestrado]. Universidade Estadual do Ceará, Fortaleza; 2014

12. Barros ML. Construção e validação de uma cartilha educativa sobre cuidados no perioperatório da cirúrgia bariátrica. [Dissertação mestrado]. Universidade Federal do Ceará, Fortaleza; 2015.

13. Galdino YLS. Construção e validação de cartilha educativa para o autocuidado com os pés de pessoas com diabetes. [Dissertação Mestrado]. Universidade Estadual do Ceará, Fortaleza; 2014. 
14. Lima ACMACC, Bezerra KC, Sousa DMN, Rocha JF, Oriá MOB. Construção e Validação de cartilha para prevenção da transmissão vertical do HIV. Acta Paul Enferm. 2017; 30(2):181-9.

15. Gentil LLS. Tecnologia educativa de cuidados para o pósoperatório da cirurgia de revascularização miocárdica: uma ferramenta para o paciente e família. [Dissertação mestrado]). Universidade de São Paulo, São Paulo; 2013.

\section{Como citar este artigo:}

Cardoso IBP, Marinho DF. Tecnologia educativa para o autocuidado de pacientes submetidos ao dreno torácico. Rev. Aten. Saúde. 2019; 17(61): 74-82. 